\title{
Symmetry Energy as a Function of Density and Mass
}

\author{
Pawel Danielewicz and Jenny Lee \\ National Superconducting Cyclotron Laboratory and Department of Physics and Astronomy, \\ Michigan State University, East Lansing, Michigan 48824, USA
}

\begin{abstract}
Energy in nuclear matter is, in practice, completely characterized at different densities and asymmetries, when the density dependencies of symmetry energy and of energy of symmetric matter are specified. The density dependence of the symmetry energy at subnormal densities produces mass dependence of nuclear symmetry coefficient and, thus, can be constrained by that latter dependence. We deduce values of the mass dependent symmetry coefficients, by using excitation energies to isobaric analog states. The coefficient systematic, for intermediate and high masses, is well described in terms of the symmetry coefficient values of $a_{a}^{V}=(31.5-33.5) \mathrm{MeV}$ for the volume coefficient and $a_{a}^{S}=(9-12) \mathrm{MeV}$ for the surface coefficient. These two further correspond to the parameter values describing density dependence of symmetry energy, of $L \sim 95 \mathrm{MeV}$ and $K_{\text {sym }} \sim 25 \mathrm{MeV}$.
\end{abstract}

Keywords: symmetry energy, mass formula, nuclear matter

PACS: 21.10.Dr, 21.65.+f

\section{INTRODUCTION}

Symmetry energy is first encountered in nuclear physics in the empirical nuclear energy formula as the term describing drop in nuclear binding with increasing neutron-proton asymmetry. That term is quadratic in the asymmetry, expressing the charge symmetry, symmetry of nuclear interactions under neutron-proton interchange. In nuclear matter, the energy as a function of neutron and proton densities may be expanded in the matter asymmetry. Knowledge of density dependence of the expansion coefficient and of the energy of symmetric matter suffices in practice for the determination of nuclear energy and pressure at any density and asymmetry, which is essential for calculations of neutron star structure [1]. The coefficients of expansion for finite nuclei and nuclear matter are connected in a nontrivial manner.

Specifically, the density dependence of the symmetry-energy expansion coefficient in nuclear matter gets tied to a variation with mass of the symmetry-energy coefficient in the empirical energy formula, due to changing contributions to the energy of nuclear volume and of surface where nuclear density drops. As a consequence, it may be possible to constrain the density-dependence of symmetry energy using the mass-dependence of the symmetry coefficient. In the following, we deduce the symmetry energy coefficient as a function of nuclear mass, using nuclear energies. Moreover, we employ Skyrme-HartreeFock (SHF) calculations for half-infinite matter and for spherical nuclei, combined with Hohenberg-Kohn functional theory, to constrain the density-dependence of symmetry energy. 


\section{SYMMETRY COEFFICIENT AND ISOVECTOR DENSITY}

Considerations [2, 3] of the competition between nuclear surface and volume in storing neutron-proton asymmetry leads to the following formula for nuclear energy

$$
E(N, Z)=-a_{V} A+a_{S} A^{2 / 3}+\frac{a_{a}(A)}{A}(N-Z)^{2}+a_{C} \frac{Z^{2}}{A^{1 / 3}}+E_{\mathrm{mic}}
$$

where $E_{\text {mic }}$ represents microscopic contributions to the energy and where the massdependent symmetry coefficient $a_{a}(A)$ follows from

$$
\frac{A}{a_{a}(A)}=\frac{A}{a_{a}^{V}}+\frac{A^{2 / 3}}{a_{a}^{S}} .
$$

In the above, $a_{a}^{V}$ and $a_{a}^{S}$ are the volume and surface symmetry coefficients and Eq. (2) states that the capacity of the system for asymmetry is a sum of the interior and surface capacitances.

The energy per nucleon in nuclear matter, expanded in the relative asymmetry $\eta=$ $\left(\rho_{n}-\rho_{p}\right) / \rho$, becomes

$$
\frac{E}{A}=\frac{E_{0}}{A}(\rho)+\frac{E_{a}}{A}(\rho, \eta) \simeq \frac{E_{0}}{A}(\rho)+S(\rho) \eta^{2} .
$$

The coefficient $S$ is typically expanded around the normal density as

$$
S(\rho)=a_{a}^{V}+\frac{L}{3 \rho_{0}}\left(\rho-\rho_{0}\right)+\frac{K_{\mathrm{sym}}}{18 \rho_{0}^{2}}\left(\rho-\rho_{0}\right)^{2}+\ldots
$$

Considerations [4] of the nuclear Hohenberg-Kohn (HK) energy functional [5], smoothed out to make it analytic, imply that the mass-dependent symmetry coefficient may be generally represented as

$$
\frac{A}{a_{a}(A)}=\frac{1}{a_{a}^{V}} \int \mathrm{d} \boldsymbol{r} \rho_{a}(\boldsymbol{r})
$$

where the isovector density $\rho_{a}$ is defined as

$$
\rho_{a}(\boldsymbol{r})=\frac{\rho_{n}(\boldsymbol{r})-\rho_{p}(\boldsymbol{r})}{\eta_{V}}
$$

Here, $\eta_{V}$ is asymmetry in the interior of matter.

In the limit of weak Coulomb effects, the isovector and isoscalar densities change only within the second order in asymmetry across an isobaric chain [4]. That implies that, in the continuum limit across medium and heavy nuclei, the proton and neutron densities can be in practice described in terms of just 4 parameters, radius parameter, difusenesses for isoscalar and isovector densities and the relative displacement of isovector and isoscalar densities. The last 2 parameters are tied to the density dependence of symmetry energy. 


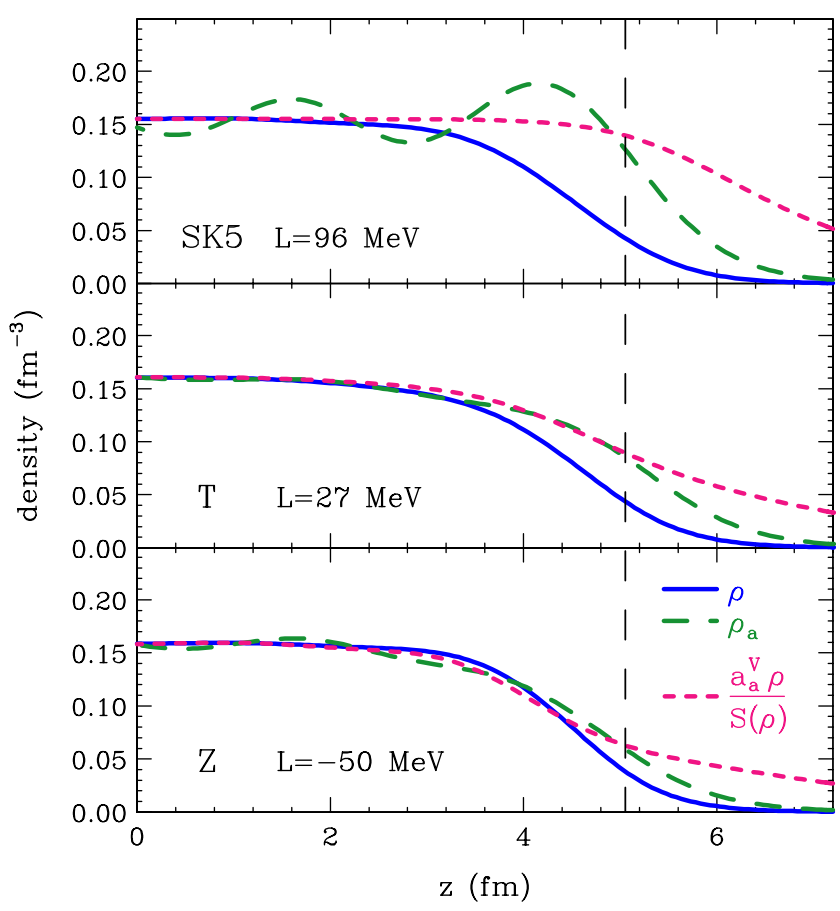

FIGURE 1. Isoscalar (solid curves) and isovector (dashed) density in half-infinite nuclear matter, together with a local approximation to the isovector density (short-dashed), as a function of position, for sample Skyrme interactions.

In the limit of short nonlocality range in the symmetry part of the HK energy functional, compared to nuclear densities, the isovector density is

$$
\rho_{a}=\frac{a_{a}^{V}}{S(\rho)} \rho
$$

In Fig. 1, we show densities from our SHF calculations of half-infinite nuclear matter. Before such calculations have been carried out Farine et al. [6]. For $\rho \gtrsim \rho / 4$, the exact isovector densities exhibit Friedel oscillations around the respective local approximations given by Eq. (7). This implies that differences between proton and neutron densities, and even proton densities alone, can directly express the density dependence of $S$ at $\rho>\rho / 4$. At $\rho \lesssim \rho / 4$, nonlocalities in the HK energy functionals become strong as evidenced in the discrepancies between exact and approximate isovector densities.

From the energy of half-infinite matter and/or vector density we can determine $a_{S}$ and $a_{a}^{S}$ coefficients in the energy formula (1) for the Skyrme interactions. While the surface coefficients, $a_{S} \sim 19 \mathrm{MeV}$, vary little between different interactions, the surface symmetry coefficients $a_{a}^{S}$ vary widely, from 9 to $75 \mathrm{MeV}$. The coefficients $a_{a}^{S}$ are tightly correlated to the symmetry-energy slope parameter $L$, see Fig. 2. A correlation also is found between scaled $L$ and $K_{\text {sym }}$. Those correlations are likely due to the lack of other energy scale in the energy functional than $\rho_{0}$. 


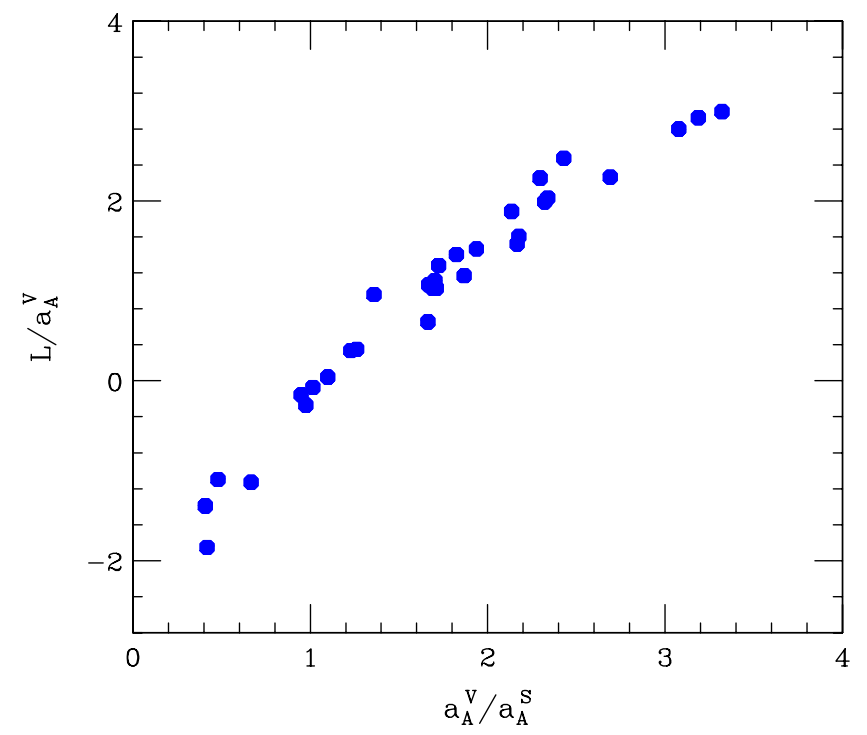

FIGURE 2. Correlation between the slope parameter $L$ and inverse of the surface symmetry parameter $a_{a}^{S}$, for Skyrme interactions from the compilation by Stone [7]. The parameters are scaled with $a_{a}^{V}$.

\section{$a_{a}(A)$ FROM ISOBARIC ANALOG STATES}

Competition between different physics terms within an energy formula hampers the ability to learn about mass dependence of the nuclear symmetry coefficient by fitting directly a formula to the ground-state nuclear energies [3, 8]. However, the unwanted competition may be practically eliminated by generalizing the energy formula to lowest states of given net isospin within a nucleus [9], amounting to the replacement of the symmetry term in (11):

$$
a_{a}(A) \frac{(N-Z)^{2}}{A}=4 a_{a}(A) \frac{T_{z}^{2}}{A} \Rightarrow 4 a_{a}(A) \frac{T(T+1)}{A} .
$$

This replacement absorbs the so-called Wigner term from $E_{\text {mic }}$. With the formula generalization, it becomes possible to deduce the symmetry coefficient within a single nucleus, by using excitation energies to the isobaric analog states (IAS) representing ground states of neighboring nuclei [8], with

$$
\Delta E=4 a_{a}(A) \frac{\Delta(T(T+1))}{A}+\Delta E_{\text {mic }} .
$$

We use the tabulated energies of isobaric analog states [10] and microscopic corrections to energies by Koura et al.[11] to deduce symmetry coefficients for individual nuclear masses. Those corrections include deformation effects. Deduced coefficient values range from $a_{a} \sim 9 \mathrm{MeV}$ for light $A<10$ nuclei to $a_{a} \sim 22.5 \mathrm{MeV}$ for $A>200$. Figure 3 shows inverse coefficient values plotted against inverse cube root of mass number. For $A>20$, the value systematic is approximately linear, as expected from Eq. (2). A fit with Eq. (2), produces coefficient values of $a_{a}^{V}=32.9 \mathrm{MeV}$ and $a_{a}^{S}=11.3 \mathrm{MeV}$. Similar 


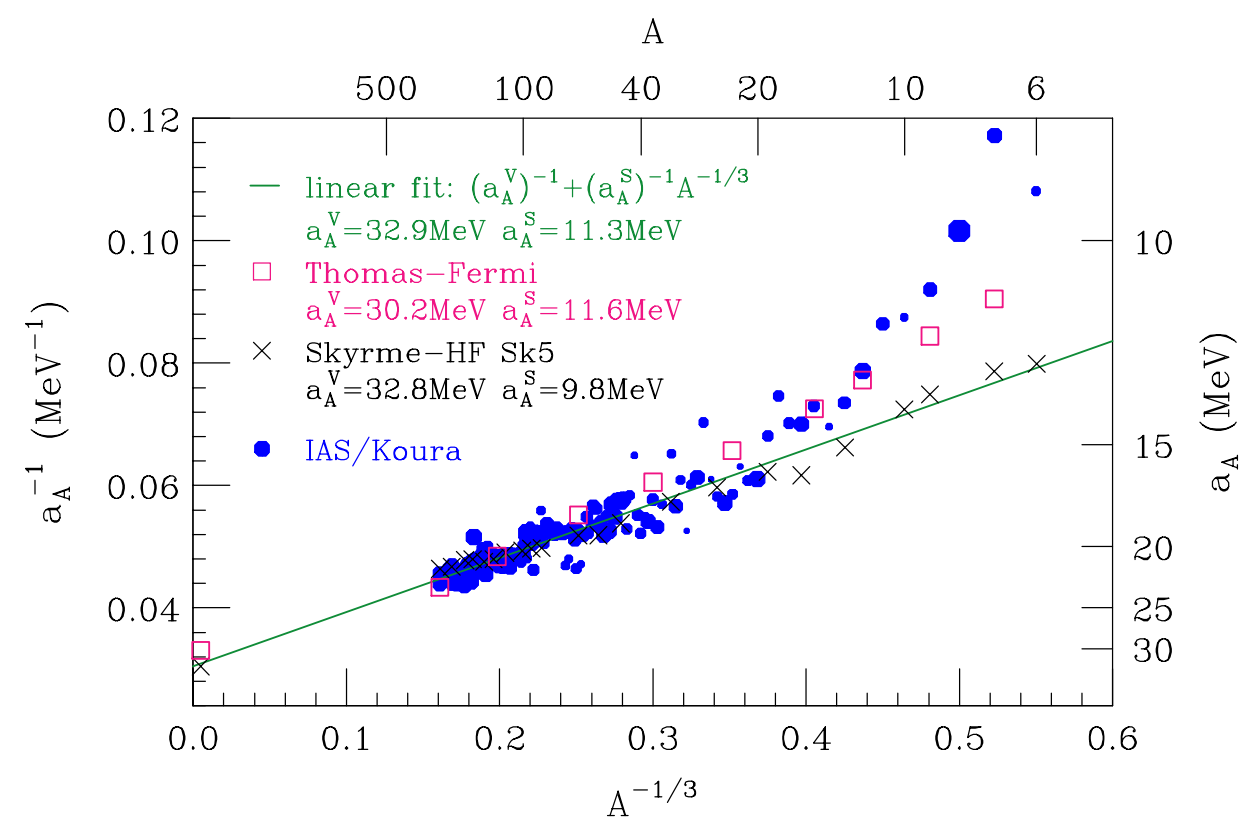

FIGURE 3. Inverse of asymmetry coefficient, in the left scale, as a function of inverse cube root of mass number, in the bottom scale. For convenience, top and right scale show, additionally, the mass number and the coefficient before inversion. The filled circles represent coefficients extracted from IAS excitation energies with the microscopic corrections applied. The line shows a fit to the IAS data for $A>20$. The crosses and squares represent, respectively, the coefficients from spherical Sk5 SHF calculations and coefficients from a Thomas-Fermi model that best describes the data.

coefficient values are obtained when trying to describe the $a_{a}(A)$ data in terms of the Thomas-Fermi theory [3, 8] or spherical SHF [12].

The parameters arrived at in different ways are further summarized in Fig. 4. The Thomas-Fermi results might be less reliable than other, because model yields an evolution of density profile in the surface region with mass, which is not fully supported by electron scattering data. Overall, the IAS data suggest parameter values of $a_{a}^{V}=(31.5-33.5) \mathrm{MeV}$ for the volume coefficient and $a_{a}^{S}=(9-12) \mathrm{MeV}$. Using the correlations between $a_{a}^{V}$ and $a_{a}^{S}$ coefficient values and $L$ and $K_{\text {sym }}$, such as in Fig. 2 , within the Skyrme model, we further arrive at values of $L \sim 95 \mathrm{MeV}$ and $K_{\text {sym }} \sim 25 \mathrm{MeV}$. These represent a nearly linear $S(\rho)$ at $\rho \lesssim \rho_{0}$.

Further efforts in this investigation strive to understand better the Coulomb effects for finite nuclei [8] and to exploit skin sizes as well as details in $\rho_{p}(r)$ for further constraining $S(\rho)$ at $\rho<\rho_{0}$.

\section{ACKNOWLEDGMENTS}

This work has been supported by the U.S. National Science Foundation under Grant Nos. PHY-0245009, PHY-0555893 and PHY-0606007. 


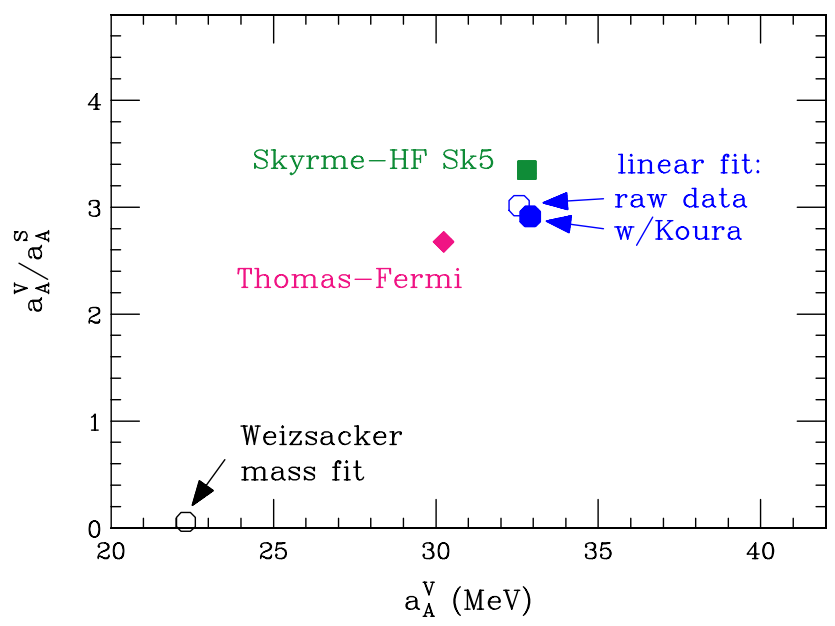

FIGURE 4. Symmetry-energy parameter values in the plane of $a_{a}^{V} / a_{a}^{S}$ and $a_{a}^{V}$, from comparing theoretical expectations to data.

\section{REFERENCES}

1. J. M. Lattimer, and M. Prakash, Phys. Rept. 442, 109-165 (2007), astro-ph/0612440.

2. W. D. Myers, and W. J. Swiatecki, Ann. Phys. 55, 395-505 (1969).

3. P. Danielewicz, Nucl. Phys. A727, 233-268 (2003), nucl-th/0301050.

4. P. Danielewicz, and J. Lee, Symmetry energy I: Semi-infinite matter in Skyrme-Hartree-Fock approach (2007), in preparation.

5. P. Hohenberg, and W. Kohn, Phys. Rev. 136, B864 (1964).

6. M. Farine, J. Côte, and J. M. Pearson, Nucl. Phys. A 338, 86-96 (1980).

7. J. Rikovska Stone, J. C. Miller, R. Koncewicz, P. D. Stevenson, and M. R. Strayer, Phys. Rev. C 68, 034324 (2003).

8. P. Danielewicz (2004), unpublished, nucl-th/0411115,

9. J. Jänecke, T. W. O’Donnell, and V. I. Goldanskii, Nucl. Phys. A 728, 23-51 (2003).

10. P. A. Antony M S, and B. J, Atomic Data and Nuclear Data Tables 66, 1-63 (1997).

11. H. Koura, T. Uno, T. Tachibana, and M. Yamada, Prog. Theor. Phys. 113, 305-325 (2005).

12. P.-G. Reinhard, "The Skyrme-Hartree-Fock Model of the Ground State," in Computational Nuclear Physics 1: Nuclear Structure, edited by K. Langanke, J. A. Maruhn, and S. E. Koonin, SpringerVerlag, New York, 1991, pp. 29-50. 